\title{
Matching rules for quasicrystals: the composition-decomposition method
}

\author{
Franz Gähler \\ Theoretical Physics, University of Geneva, 24, quai Emest Ansermet, CH-121/ Geneva 4, Switzerland
}

\begin{abstract}
A general method is presented which proves that an appropriately chosen set of matching rules for a quasiperiodic tiling enforces quasiperiodicity. This method, which is based on self-similarity, is formulated in general terms to make it applicable to many different situations. The method is then illustrated with two examples, one of which is a new set of matching rules for a dodecagonal tiling.
\end{abstract}

\section{Introduction}

With the discovery of more and more thermodynamically stable, perfect quasicrystal systems it has become unlikely that the formation of quasicrystals is due to a purely entropic effect, as had been assumed in random tilings models [1]. At least for some of the recently discovered F-type icosahedral quasicrystals it seems necessary to include, besides entropic effects, also an energetic mechanism favouring quasiperiodic arrangements of atoms in order to understand the high perfection of these quasicrystals. The simplest models with interactions favouring quasiperiodicity are tiling models, in which the atoms are assumed to form stable clusters, represented by tiles, and the residual interactions between the clusters are such that they favour a quasiperiodic arrangement of the clusters. In order that these inter-cluster interactions be effective, they must be sufficiently strong, which is in contrast to random tiling models, where one assumes that they are very weak so that they can be neglected at high temperature.

In the following, we shall deal only with tilings

Correspondence to: Dr F. Gähler, Theoretical Physics, Universily of Geneva, 24, quai Ernest Ansermet, CH.1211 Geneva 4, Switzerland. E-mail: gaehler@sc2a.unige.ch. and interactions between tiles ('matching rules'). We shall present a general method to prove that a given set of local interactions or matching rules enforces a quasiperiodic ground state. Whether or not such a quasiperiodic ground state remains stable at positive temperature is still under debate and will not be further discussed. Our proof is based on the self-similarity present in all tilings applied to the description of quasicrystals so far. The various arguments of our proof are not new; they have been used in different combinations by other authors as well, e.g. by De Bruijn [2] for the Penrose tiling. Rather, the aim of this paper is to put together all the necessary ingredients of the proof and to state them in general terms, in order to provide a method that works in many cases. Due to lack of space, many of our arguments can only be sketched, and we must refer to a future publication for further details.

The remainder of the paper is organised as follows. In section 2, the strategy of the proof is described in general terms, providing all the necessary ingredients. The proof is then illustrated with two examples, in section 3 with Ammann's well known matching rules for the octagonal square-rhombus-tiling, and in section 4 with a new set of matching rules for a dodecagonal tiling. In section 5 we conclude with some final remarks. 


\section{The general procedure}

In this section we describe the general strategy of the proof. Suppose that a set of prototiles is given, together with appropriate local rules for joining these prototiles. Typically, these rules are expressed in terms of markings of the prototiles that must match. Since we are going to use the self-similarity present in the tilings admitted by the matching rules, this self-similarity must be defined in terms of the prototiles. We assume that a decomposition rule is given, according to which each prototile is disected in a unique way into similar tiles smaller by a factor $\lambda$. These smaller tiles carry again the markings defining the matching rules. The decomposition must respect the matching rules: tiles that match must have decompositions that match. The decomposition rule allows us to construct tilings covering larger and larger areas, eventually even the whole plane, by repeatedly applying the decomposition step and enlarging the resulting tiling each time by a factor $\lambda$, to get back to the original tile size.

Let us now explore the set of all tilings admitted by the matching rules. We do this by a three step procedure. The first step has been described in detail in ref. [3] and serves to prove that the matching rules enforce non-periodicity. We note that every global tiling admitted by the matching rules has a unique decomposition which is again admitted by the matching rules. The idea is to prove that there exists a unique inverse operation, called composition. That is, we must prove that for each tiling there exists a unique tiling larger by a factor $\lambda$ of which the current tiling is the decomposition. For that, two things must be shown, namely: (i) given any tiling admitted by the matching rules, each of its tiles can be composed, together with part of its neighbourhood, to a unique 'supertile'. This supertile may depend on the neighbourhood, but the uniqueness makes sure that no contradiction arises when the supertiles for different tiles are constructed. (ii) The markings which the supertiles inherit from the small tiles must enforce matching rules that are equivalent to those of the corresponding tile of the original size. If these two points are satisfied we can show that the matching rules enforce non-periodicity. To see this enforcement, suppose that we have a tiling left invariant by some translation. We can now apply the composition procedure to this tiling. Due to the uniqueness of composition, no information is lost in this process. With each composition step, the period length, expressed in the scale of the current tile size, shrinks by a factor $\lambda$, until it is so small that peridodicity can be ruled out by inspection of all possible local neighbourhoods.

The procedure described above is a standard method [3] to prove that a set of matching rules enforces non-periodicity. So far, however, we have not learned anything on the possible quasiperiodicity of the tilings admitted by the matching rules. Therefore, in a second step, we shall describe how to construct such tilings, including the markings, by a method that guarantees their quasiperiodicity, namely the projection method [4]. In the projection method, the vertices of the tiling are projections of certain nodes of a higher dimensional lattice onto a subspace, called physical space. All those lattice nodes are projected which fall into an appropriate window region in the orthogonal complement of physical space. Rules are set for joining vertices to form tiles, and how tiles, bonds or vertices have to be marked. In order to maintain quasiperiodicity, these rules may depend only on where in the window the corresponding objects fall. There are uncountably many different tilings obtained in this way, depending on the position of the window relative to the lattice. If we can find such a projection method which produces tilings with the correct and correctly decorated tiles, such that the decorations of neighbouring tiles match, we have an infinity of quasiperiodic tilings admitted by the matching rules.

In a third step, we have to show that there are no other tilings admitted by the matching rules. We shall again use the self-similarity, which we therefore must define also for the projection tilings. This can be done in a global way [5]. Each tiling has a unique deflation and inflation, as decomposition and composition are called in this context. Both the deflation and the inflation of a tiling get their markings from the projection method. All we have to do is to check that 
decomposition and deflation on the one hand and composition and inflation on the other hand exactly agree. Given any tiling admitted by the matching rules, we can now construct a sequence of quasiperiodic projection tilings which agree with it on larger and larger areas. To show that there exists a projection tiling which agrees with the given tiling in a ball of radius $R$, we repeatedly apply the composition procedure to it ( $n$ times), until the original ball of radius $R$ is contained in some vertex neighbourhood of the composed tiling. Then we take a projection tiling containing the same vertex neighbourhood, and apply to both tilings $n$ times the decomposition and deflation procedure, respectively, which reproduces the original tiling, plus a projection tiling which agrees with it on a ball of radius $R$. In this way, any tiling admitted by the matching rules can be obtained as a limit of projection tilings. It can be shown that these limits are projection tilings themselves, so that we can prove that actually all tilings admitted by the matching rules are quasiperiodic projection tilings.

\section{Ammann's octagonal tiling}

As a first example we consider Ammann's matching rules for the octagonal tiling $[3,6]$. In fig. 1, a piece of such a tiling is shown, and one can see the constituant tiles with their markings. Underlaid in gray are the markings of its composition. The matching rules demand that the halfcircles on the edges match, and that the markings at the vertices form arrows. Socolar [6] has already proved that these rules enforce quasiperiodicity, using the concept of Ammann bars. We present here an independent proof, which seems somewhat more transparent.

The first step in our procedure has already been done in ref. [3], so that we will not repeat it here. We directly pass to step two and present how the markings are obtained in the projection method. The window region is an octagon, as shown in fig. 2 , and the markings are chosen as follows: the octagon is divided by four lines through its center which are perpendicular to the possible bonds. The orientation of a bond, indi-

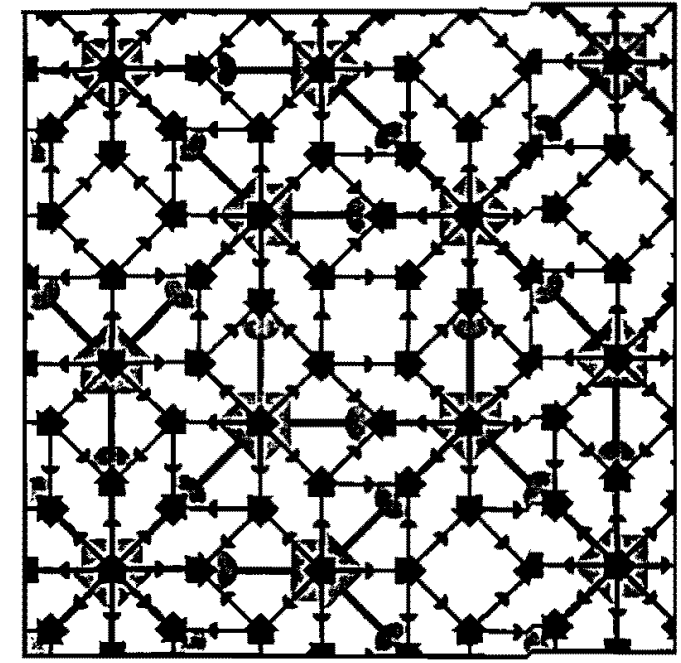

Fig. 1. A piece of Ammann's octagonal tiling with markings defining the matching rules. Its composition is underlaid in gray.

cated by the half-circle, is chosen according to whether the mid-point of the bond is left or right of the corresponding line. Furthermore, the octagon is divided into eight sectors, such as the shaded one. To each sector there corresponds an orientation of the arrow on a vertex. It is easy to verify that with such an assignment all the tiles are correctly decorated, and by construction the decorations of neighbouring tiles must match. It is also straightforward to check that deflation and inflation for the projection tiling agree with com-

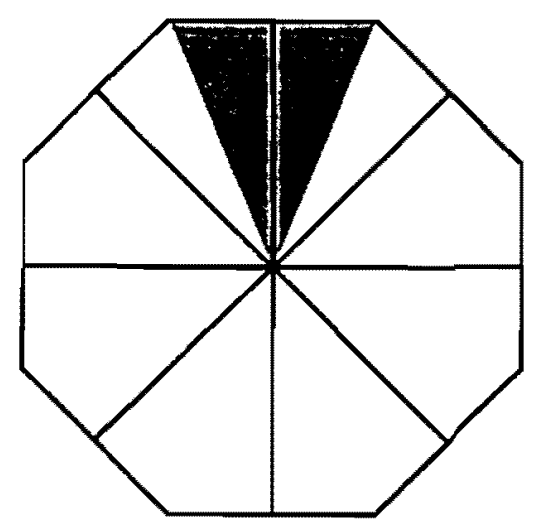

Fig. 2. Subdivision of the window region for Ammann's octagonal tiling. 


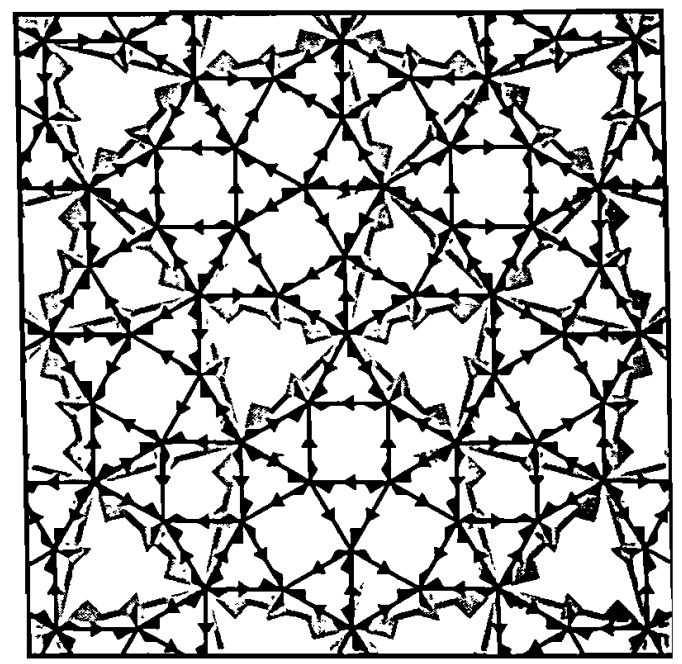

Fig. 3. A piece of a dodecagonal tiling with markings defining a new set of matching rules. Its composition is underlaid in gray.

position and decomposition as given by Ammann [3], so that we can apply the reasoning of step three. The only subtle point is that there are so called singular (projection) tilings, for which there are vertices or bond mid-points falling on the boundaries between the different subregions of the window, so that the decoration is not unique. This ambiguity can be resolved by an infinitesimal translation of all vertices, although not in a unique way, so that in such a case we obtain several different tilings, all with a valid decoration. These singular tilings can, however, be obtained as limits of regular tilings.

\section{A new dodecagonal tiling}

As a second example we present a new set of matching rules for a dodecagonal tiling, a piece of which is shown in fig. 3 . The tiling consists of squares, triangles and threefold symmetric hexagons. The markings defining the matching rules and the decomposition rules are indicated in fig. 3. The triangle may have two different markings, and each tile comes in a left handed and in a right-handed version. The matching rules demand that arrows on edges match, and that the markings at the vertices form a 'cross'. Note that there are left crosses and right crosses. With arguments similar to those in ref. [3], it is not very difficult to show that a unique composition exists, but we do not have the space to go into details. The essential point is that a change of scale by $\lambda=\sqrt{2+\sqrt{3}}$ is chosen [5], and not $\lambda=2+\sqrt{3}$, which simplifies the task considerably. The projection method to construct this tiling has been given in ref. [7]. Its window region is a dodecagon, shown in fig. 4 with the subdivisions corresponding to the different markings. As with Ammann's tiling, the lines decide on the orientation of the bonds, and the shaded sector corresponds to one orientation of the cross. The line which divides the shaded sector separates regions corresponding to left crosses and right crosses of a given orientation. It is straigthforward to check that these assignments lead to the markings and decomposition rules shown in fig. 3 , so that we can apply the standard reasoning of section 2 . Singular tilings are dealt with as in section 3 . The tiling presented here is closely related to another dodecagonal tiling, given by Socolar [6]. The undecorated versions of these tilings can be locally derived from each other [8]. The same happens to be true for the decorated tilings, although our matching rules have been found independently. By superimposing Socolar's Ammann bars on our tiling it is not difficult to see that they impose

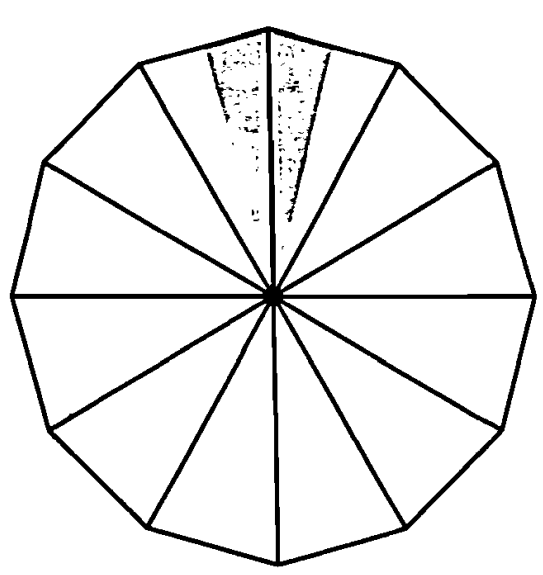

Fig. 4. Subdivision of the window region for the dodecagonal tiling. 
matching rules equivalent to ours, which provides a alternate proof for Socolar's matching rules [6].

\section{Concluding remarks}

We have presented a general method to prove that a set of matching rules enforces quasiperiodicity. This method can work, of course, only if the matching rules are correctly chosen from the very beginning. Unfortunately, we do not have a method to find the correct matching rules for a given tiling.

\section{References}

[1] See e.g.: C.L. Henley, in: Quasicrystals: The State of the Art, eds. PJ. Steinhardt and D.P. DiVincenco (World Scientific, 1991) p. 429.

[2] N.G. de Bruijn, Ned. Akad. Wetensch. Proc. Ser. A43 (1981) 39, 53.

[3] R. Ammann, B. Grünbaum and G.C. Shephard, Discrete Comput. Geom. 8 (1992) 1.

[4] F. Gähler and J. Rhyner, J. Phys. A19 (19B6) 267.

[5] K. Niizeki, J. Phys. A22 (1989) 193.

[6] J.E.S. Socolar, Phys. Rev. B39 (1989) 105 ]9.

[7] F. Gähier, in: Quasicrystalline Materials, eds. Ch. Janot and J.M. Dubois (World Scientific, 1988) p. 272.

[8] H.-U. Nissen, in: Quasicrystals, Networks, and Molecules of Fivefold Symmetry, ed. 1. Hargittai (VCH Publishers, 1990) p. 181. 